\title{
LIMITATION OF THE PUPIL REPLICATION TECHNIQUE IN THE PRESENCE OF INSTRUMENTAL DEFECTS
}

\author{
P. Riaud, ${ }^{1}$ D. Mawet, ${ }^{1}$ and O. Absil ${ }^{1}$ \\ Institut d'Astrophysique et de Géophysique de Liège, 17 Allée du 6 Août, Bât B5c, B-4000 Liège, Belgium; \\ riaud@astro.ulg.ac.be, mawet@astro.ulg.ac.be, absil@astro.ulg.ac.be \\ Received 2005 February 25; accepted 2005 June 7; published 2005 July 5
}

\begin{abstract}
Pupil replication has been proposed by Greenaway et al. as a new optical technique to improve the suppression of starlight in high dynamic imaging. This Letter extends numerical simulations in the two-dimensional case with various realistic imperfections (surface error, chromatic smearing, and pupil shift). These results demonstrate some strong limitations compared to single-pupil apodization techniques for exoplanet detection.
\end{abstract}

Subject headings: planetary systems — stars: imaging — techniques: high angular resolution

\section{INTRODUCTION}

The main problem in the search for exoplanets is the contrast between the host star and its potential exoplanets. For an Earthlike planet, the flux ratio is $10^{10}$ in the visible and $10^{6}$ in the thermal infrared around $10 \mu \mathrm{m}$. Generally, the star halo strongly contaminates planetary images. The Airy rings can be attenuated by appropriate pupil apodization techniques (Aime et al. 2002), for example. But far away (beyond $10 \lambda / d$, with $d$ being the telescope diameter), the ultimate speckle level limits the detectability of faint sources such as exoplanets. Greenaway et al. (2005, hereafter GR05) have recently presented a new technique called "pupil replication" to decrease the diameter of the star image in the focal plane. This technique transforms a simple telescope into a pseudointerferometer by juxtaposing a number of replicas of the initial pupil. Therefore, the field of view properties are analogous to the pupil densification technique (Labeyrie 1996; Pedretti et al. 2000; Riaud et al. 2002; Gillet et al. 2003). A classical apodization scheme (super-Gaussian) is presented in GR05 to achieve adequate suppression of the stellar halo. In this Letter, unlike in GR05 where all simulations are one-dimensional, we study the optical properties for two-dimensional systems with the following consequences: classical circular apertures do not appear optimal for the pupil replication technique, and only pupil shapes like squares or hexagons can be used to achieve optimal extinction effects. Moreover, the two-dimensional simulations clearly show a dispersion of the planetary flux, not only in the radial direction, as in one-dimensional images, but across the whole image plane (see Fig. 3 below). The associated flux losses are thus larger than the ones observed in the one-dimensional simulations in GR05. Then, we discuss the effect of surface errors due to polishing defects in the optical workbench. Finally, the most severe source of performance degradation seems to be the possible misalignment between the subpupils, whereas pointing errors result in only weak losses. This article explains and quantifies these principal limitations of the pupil replication technique for exoplanet detection.

\section{SIMULATION RESULTS}

\subsection{Basic Results}

We have performed two-dimensional numerical simulations of pupil replication in conditions close to the ones used in GR05. The replication system consists of $3 \times 3$ pupils arranged

${ }^{1}$ Postdoctoral Astronomer, under PAI contract. on a square. With this configuration, the core of the star is 3 times narrower in the $(X, Y)$-directions than in the single-pupil case. In our simulations, all images (replicated and not replicated) correspond to a sum of 20 elementary images at different wavelengths with the appropriate (super-Gaussian) apodization technique in the considered bandpass (760-1000 nm, like in GR05). All angular separations will be given with respect to the central wavelength of $880 \mathrm{~nm}$, and we will consider that the replication scheme is perfect in terms of transmission (ideal beam splitters). In the one-dimensional case, the optical efficiency of the system is $65 \%$ for square pupils, and in the twodimensional case, it is $\approx 41 \%$. This difference is simply due to the conservation of energy (each replica possess $\frac{1}{9}$ of the total energy) and the two-dimensional geometry integration: the apodization profile must be azimuthally integrated on the $3 \times 3$ replicated pupil, which leads to a very poor transmission for the four replicas at the corners of the scheme.

Three possible configurations can be considered with circular, square, or hexagonal subpupils. The first configuration (circular), although the most common, is unfortunately not optimized for the best rejection factor due to the gaps between replicated pupils. The last two configurations (square and hexagonal) produce a replicated arrangement without gaps; thus, they are optimized for high-contrast imaging. In the following study, we will therefore focus on the square-pupil geometry with the super-Gaussian apodization. ASA apodization (Nisenson \& Papaliolos 2001) appears to work best for the squarepupil geometry, but unfortunately it is also more difficult to manufacture. In order to have a full working angle and to provide a direct comparison with GR05, the chosen apodization (super-Gaussian) is centrosymmetric.

The first simulations consist in comparing the results in the one- and two-dimensional cases (the latter not being considered in GR05). With the square geometry, the best result in the residual level is $10^{-14}$ of the central star flux. We will see in the following section that this level is critically dependent on the wave-front bumpiness and on pupil misalignments. Figure 1 compares the replicated apodized case with a single apodized pupil. The numerical simulations are performed with a low wavefront error $(\lambda / 2500 \mathrm{rms}$ at $632.8 \mathrm{~nm})$ and in polychromatic light (760-1000 nm).

\subsection{Performance Estimation for a Real System}

Now let us investigate three important limitations of the pupil replication technique. First, the wave-front bumpiness of both the telescope and the optical replication scheme is considered. 


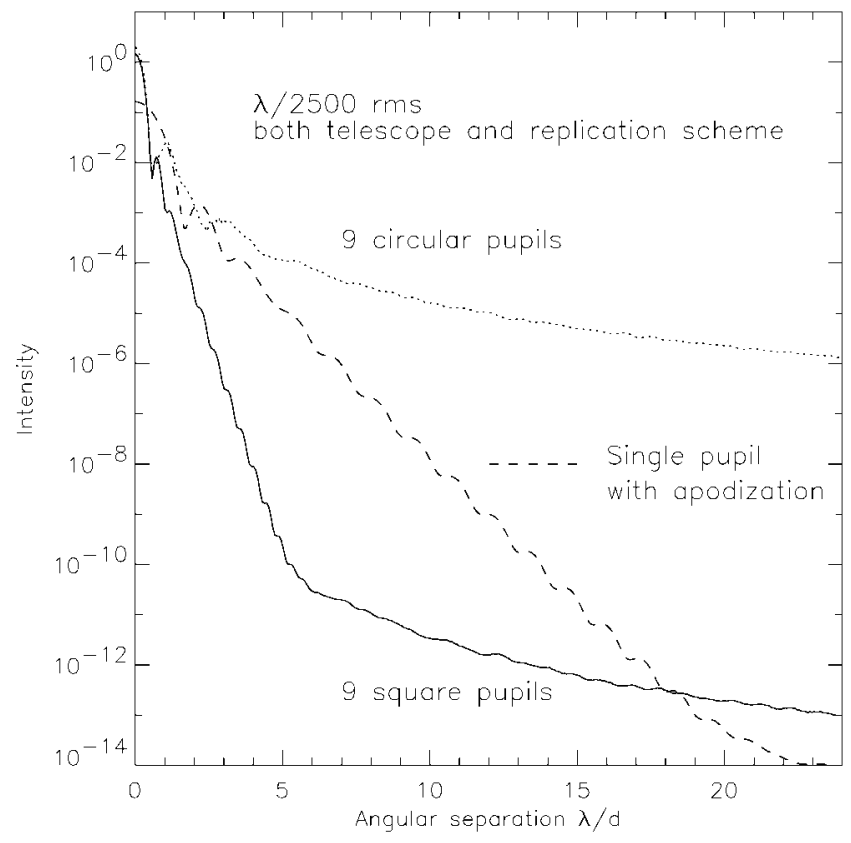

FIG. 1.-Pupil replication analysis. Numerical simulations illustrating the effect of replication on the square pupil. The solid line shows the starlight level as a function of angular distance in the case of the super-Gaussian apodization for the two-dimensional replicated scheme (nine replicated pupils). The dotted line shows the case of a two-dimensional replicated scheme with nine circular pupils. The dashed line illustrates the apodization effect on a single pupil. All simulations are performed in polychromatic $(760-1000 \mathrm{~nm})$ and low wave-front error $(\lambda / 2500 \mathrm{rms}$ at $632.8 \mathrm{~nm})$. The effect of the replication technique on the star level is clearly visible: only the domain between $1 \lambda / d$ and $18 \mathrm{~N} / d$ presents a possible gain with a square entrance pupil for high-contrast imaging. The classical circular entrance pupil presents less interest.

Indeed, we have so far presented the basic results with ultimate optics characteristics $(\lambda / 2500 \mathrm{rms}$ at $632.8 \mathrm{~nm})$. Figure 2 shows the results obtained with various wave-front errors between $\lambda / 30$ and $\lambda / 2500 \mathrm{rms}$. To simplify the analysis, we suppose that the wave-front bumpiness of the replication scheme is the same as for the telescope, which might be an optimistic supposition (the beam splitters will not be perfect). Thus, all wavefront errors mentioned in this article should be multiplied by $\sqrt{2}$ to have the global wave-front error. In Figure 2, we clearly see an important gain for wave-front errors smaller than $\lambda / 250 \mathrm{rms}$. This result is interesting in terms of feasibility. Indeed, the Virgo team (Mackowski et al. 1999; Brillet et al. 2003) has demonstrated a state-of-the-art mirror quality with excellent polishing realization $(\lambda / 226 \mathrm{rms}$ at $632.8 \mathrm{~nm})$, within the framework of gravitational wave detection. This technology is directly applicable to Bracewell nulling interferometers (Bracewell 1978), apodization, and coronagraphy (Baudoz et al. 2000; Riaud et al. 2003). Other applications, such as the EUV lithography process, also need excellent mirror quality. Let us cite, for example, the realization of small EUV mirrors with a wave-front error around $\lambda / 1800 \mathrm{rms}$ at $632.8 \mathrm{~nm}$ (Goldberg et al. 2002). The first value given by the Virgo team seems to be realistic, although somewhat pessimistic, in the sense that further developments may improve this figure. A $\lambda / 250 \mathrm{rms}$ wave-front bumpiness seems to be a reasonable goal.

The second limitation of pupil replication for high-contrast imaging is the flux attenuation of an off-axis source. The pupil replication can be seen as a pseudointerferometer creating multiple dispersed peaks referred to as "chromatic smearing" in GR05. The calculated flux attenuation of an off-axis source in four resolution elements, i.e., $(2 \lambda / d)^{2}$, is an oscillating function. Figure 3 shows numerical simulations of off-axis sources in

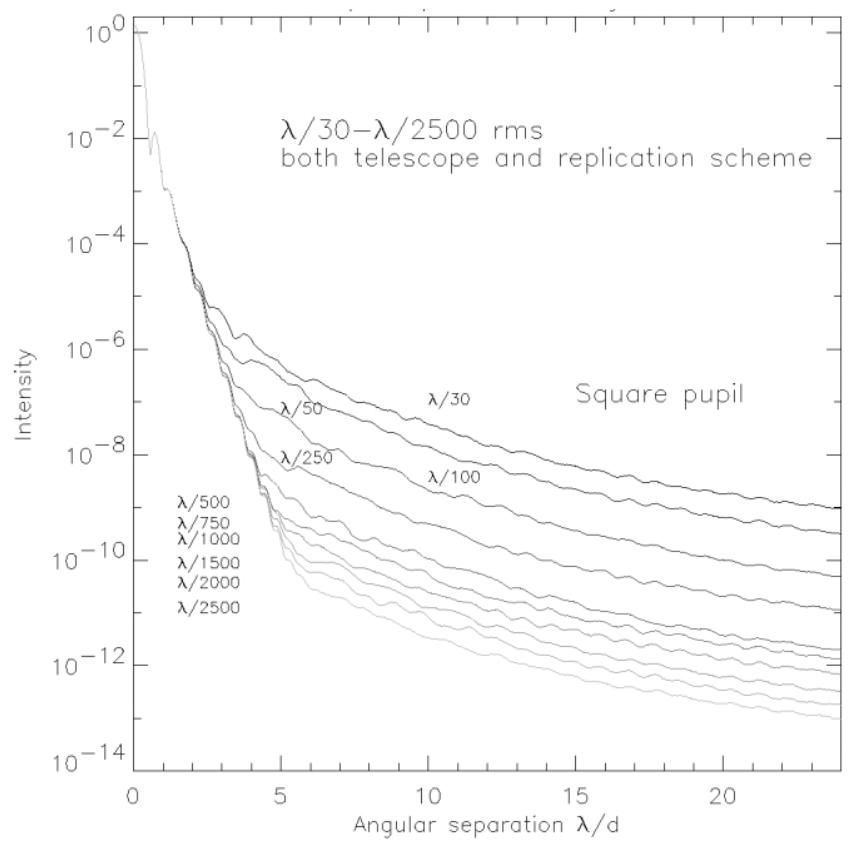

FIG. 2.-Pupil replication analysis. Simulated radial profiles for square pupil replication in the presence of polishing errors. We present the starlight level for various wave-front errors between $\lambda / 30$ and $\lambda / 2500 \mathrm{rms}$ for both the telescope and replication optics. The simulations show an important gain for wavefront errors smaller than $\lambda / 250 \mathrm{rms}$.

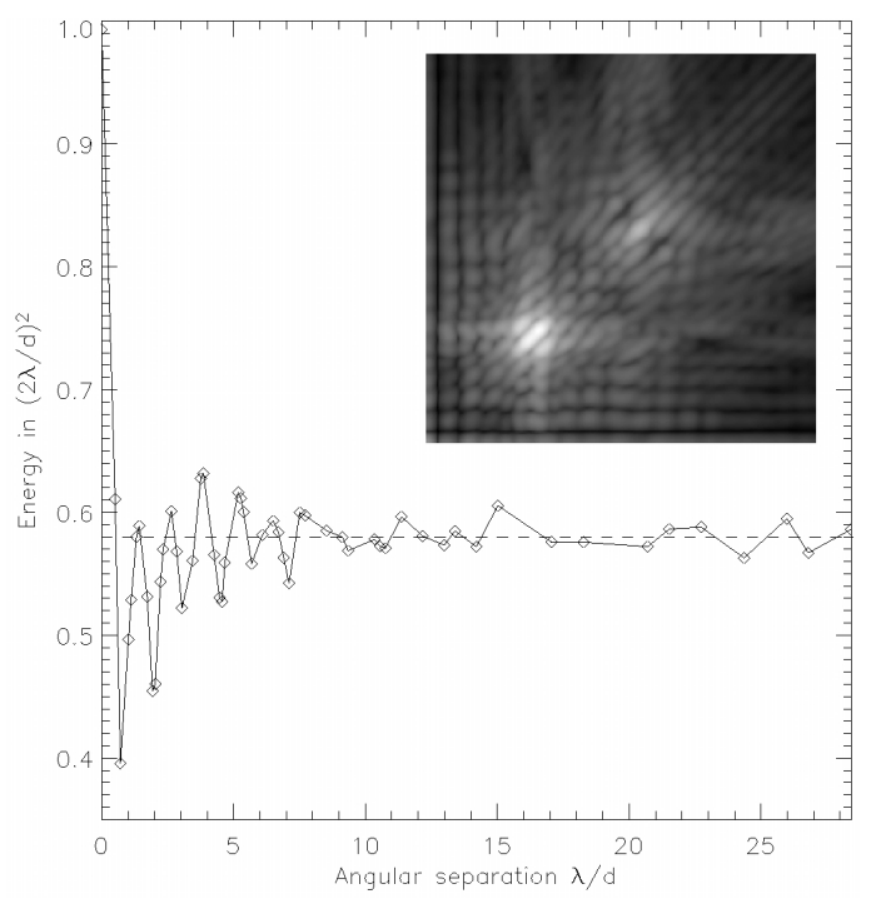

FIG. 3.-Planet attenuation. Numerical simulations of chromatic smearing of a planetary peak. An off-axis planetary peak spreads out on a large surface, thereby inducing a dilution of flux. We calculate the flux of an off-axis pointlike source in four resolution elements centered around the maximum of the intensity. We demonstrate here a flux loss depending on the angular separation. We show large oscillations for angular separations smaller than $10 \mathrm{~N} / \mathrm{d}$ and then a quasi-constant loss of $42 \%$ farther out. The image shows the response of the replication system in logarithmic scale for an off-axis planet $(4 \lambda / d)$ with the lower left corner being on-axis. 


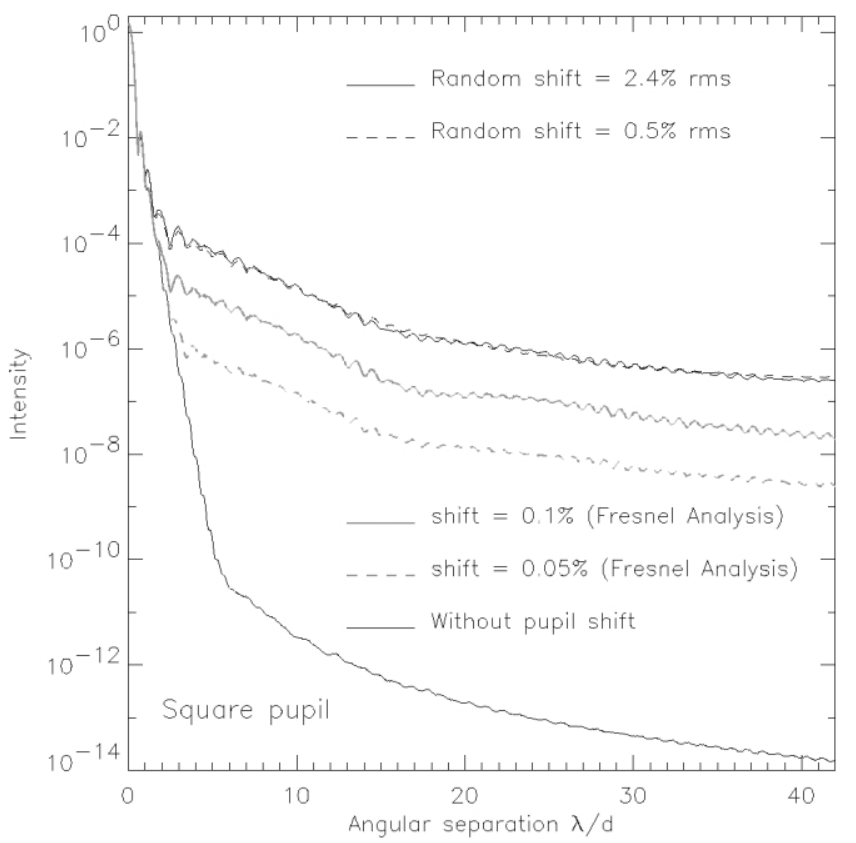

FIG. 4.-Pupil replication analysis. Simulated effect of pupil misalignment in the replication optical scheme. A $0.5 \%$ rms already gives an important loss of about 7 orders of magnitude for angular separations larger than $3 \lambda / d$. The dashed curve at $0.5 \%$ presents only small variations with respect to the first solid curve $(2.4 \%)$. This relates to the fact that the presence of gaps induces high diffraction residues in the final images. A Fresnel diffraction analysis $(0.1 \%$ and $0.05 \%$ pupil misalignment $)$ shows a significant improvement in stellar rejection by a factor 10 and 100, respectively. Assuming a $1 \mathrm{~cm}$ replicated pupil, these misalignments respectively correspond to $240,50,10$, and $5 \mu \mathrm{m}$. A $10 \mu \mathrm{m}$ alignment precision is currently state-of-the-art. The gap between each replica should be smaller than $2 \mu \mathrm{m}$ to achieve a $10^{-10}$ level of contrast at $40 \mathrm{~N} d$.

the case of $\lambda / 2500 \mathrm{rms}$ wave-front bumpiness. The mean attenuation factor is $42 \%$ with respect to the on-axis source. The attenuation in the case of $(3 \lambda / d)^{2}$ and $(\lambda / d)^{2}$ becomes $36 \%$ and $64 \%$, respectively.

A third important limitation of the new technique is the sensitivity to the relative positioning of the different replicated pupils. As already seen in Figure 1, where the pupils are not perfectly joined because of their circular shape, the starlight residuals for large angular separations can be as high as $10^{-6}$. In the case of pupil misalignments, this effect can become even more important. Indeed, if the pupil arrangement presents some gaps, the system behaves like a Fizeau interferometer and creates a large number of dispersed peaks. The large bandwidth and the scattering effect due to wave-front bumpiness give an even, increased starlight level. Figure 4 shows a loss in starlight rejection as large as 7 orders of magnitude for angular separations larger than $3 \lambda / d$ with respect to the ideal case of Figure 1, when the pupils are misaligned by $0.5 \%$ or more. A detailed analysis, including the effects of the Fresnel diffraction between the edges of the pupils (an oscillating function), shows a clear improvement in the system performance for pupil misalignments smaller than $0.1 \%$. However, assuming a $1 \mathrm{~cm}$ replicated pupil size, a good alignment with a $10 \mu \mathrm{m}$ precision still induces a loss of 6 orders of magnitude in starlight rejection.

Finally, the last effect on the residue level is the pointing jitter. Indeed, pupil replication consists in artificially increasing the angular resolution like a pseudointerferometer. Small jitter errors induce a relatively small effect compared to pupil misalignment, with a degradation ranging from a factor 4 to 6 on the whole angular domain for jitter errors between 1 and 15 mas rms. Taking all errors into account, the gain is in fact larger than 1

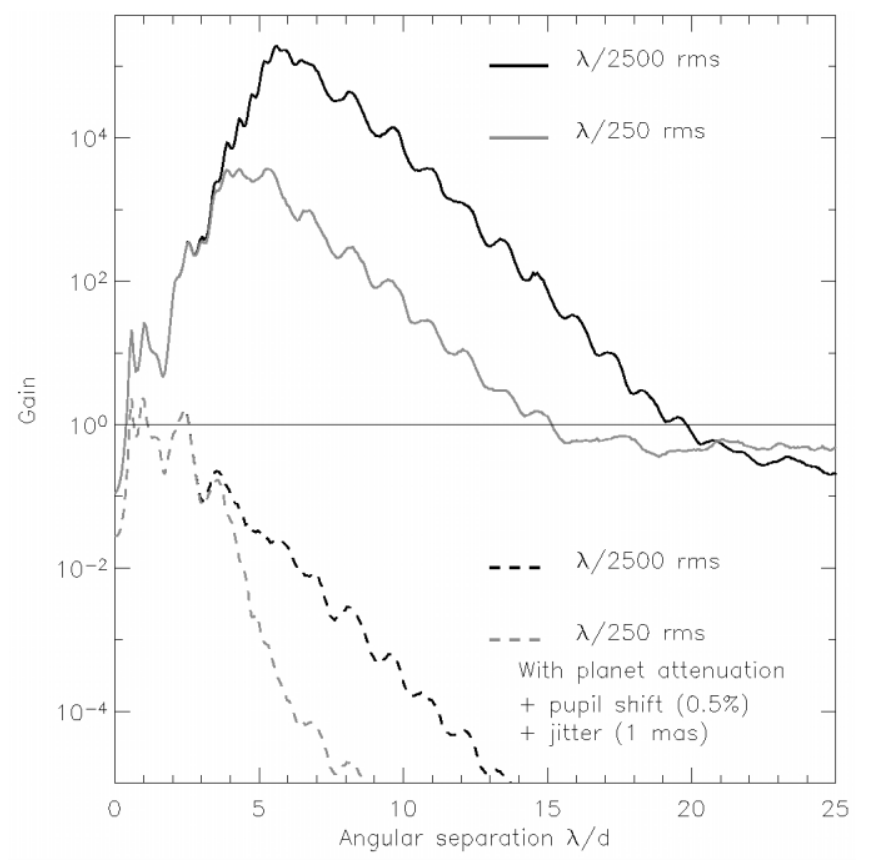

FIG. 5.-Pupil replication analysis. Numerical simulations of the gain in starlight level between the apodized and replicated schemes and the simple pupil apodization. These simulations are given for two possible wave-front bumpinesses $(\lambda / 250 \mathrm{rms}$ in gray and $\lambda / 2500 \mathrm{rms}$ in black). The two solid curves illustrate the gain provided by this technique without instrumental defects other than wave-front errors, while the two dashed curves correspond to the same numerical simulations by taking into account flux loss for an offaxis source (see Fig. 3), a pupil misalignment of $0.5 \%$ (see Fig. 4), and a 1 mas jitter. All profiles are azimuthally averaged. This simulation shows a poor gain $(\leq 5)$ for short angular separations $(<3 \lambda / d)$. With a pupil alignment around $0.1 \%$, the gain would range from 5 to 40 for angular separation lower than $<4 \lambda / d$.

only for short angular separations $(<3 \lambda / d)$ and does not exceed around 5 in the application range where the replication technique would be interesting [(1-18) $\lambda / d$; see Fig. 1], as illustrated in Figure 5. Let us emphasize that we have chosen perfect beam splitters in these simulations. With state-of-the-art commercial components $(\lambda / 100 \mathrm{rms}$ at $632.8 \mathrm{~nm}$ and an amplitude mismatch of 5\%), the total wave-front bumpiness would amount to $\lambda / 60$ rms, and the nominal performance of the system would not be achieved. A new instrumental approach is thus necessary.

\section{DISCUSSION AND PERSPECTIVES}

We have demonstrated several important limitations of the pupil replication technique presented by GR05. Originally, this technique was presented in the one-dimensional case. Our numerical analysis for the two-dimensional case shows new effects, like diffraction between adjacent subpupils due to pupil misalignment, that strongly affect the efficiency of this technique.

In the case of extreme adaptive optics implementation on ground-based telescopes or of high-contrast imaging on space telescopes, a classical Lyot coronagraph or a phase-mask coronagraph gives the same performance as this technique (or better) with a much simpler implementation and all the while preventing saturation.

The pupil replication technique must be used in tandem with other apodization or nulling schemes, not alone. It seems to be more appropriate to use this technique after a coronagraphic mask or, more precisely, after the coronagraphic diaphragm blocks most of the starlight. Doing so, we relax important limitations like wave-front bumpiness and pupil shear. The starlight diffusion induced by the wave-front defects of the replication scheme 


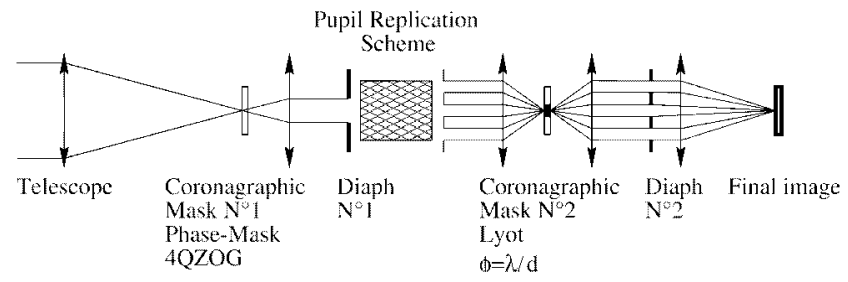

FIG. 6.-Example of multistage coronagraphic implementation of the pupil replication scheme. The first stage consists of a phase-mask coronagraph like the 4QZOG that allows a full inner working angle. The first diaphragm removes most of the starlight contribution, but even if after it, a central peak due to tip-tilt errors and chromatism still remains in the coronagraphic image. As a second stage, the replication scheme creates nine circular replicated pupils. This configuration forces the stellar peak residue to shrink in the pseudointerferometric image. In this image plane, we use as a third stage a second coronagraphic mask: a simple Lyot with a diameter of only $\lambda / d$ instead of $6 \mathrm{~N} / d$ in the classical approach of single-pupil coronagraphy. The Lyot mask removes the narrower stellar peak and improves the detectability in the final image by a factor of about 40 .

can be overcome by attenuating its main contribution upstream with a precoronagraphic stage. Thanks to the coronagraphic attenuation, the tolerance on the wave-front bumpiness is consequently relaxed at about $\lambda / 4$ PTV (peak to valley). Moreover, the upstream starlight attenuation and the absence of apodization now allow us to use circular pupils without producing a too large amount of spurious starlight in the following image plane. We propose to take advantage of these properties by using a threestage optical implementation as shown in Figure 6. The first stage consists in a phase-mask coronagraph (quasi-achromatic 4QZOG; Mawet et al. 2005) followed by a $3 \times 3$ pupil replication scheme for classical circular pupils. In the following interferometric image plane and as a third stage, we place a simple Lyot coronagraph (dark spot) followed by its diaphragm to further enhance the contrast. $K$-band preliminary numerical simulations show a gain of $\approx 40$ on the stellar peak attenuation with respect to the 4QZOG alone (Mawet et al. 2005), with a null depth of $4.5 \times 10^{-7}$. In this simulation, we take into account realistic wave-front errors for both the telescope $(\lambda / 250 \mathrm{rms})$ and the pupil replication scheme $(\lambda / 50 \mathrm{rms})$ with respect the central wavelength of $2.2 \mu \mathrm{m}$ (see Fig. 7). Nevertheless, given the optical complexity, such a multistage scheme should be ex-

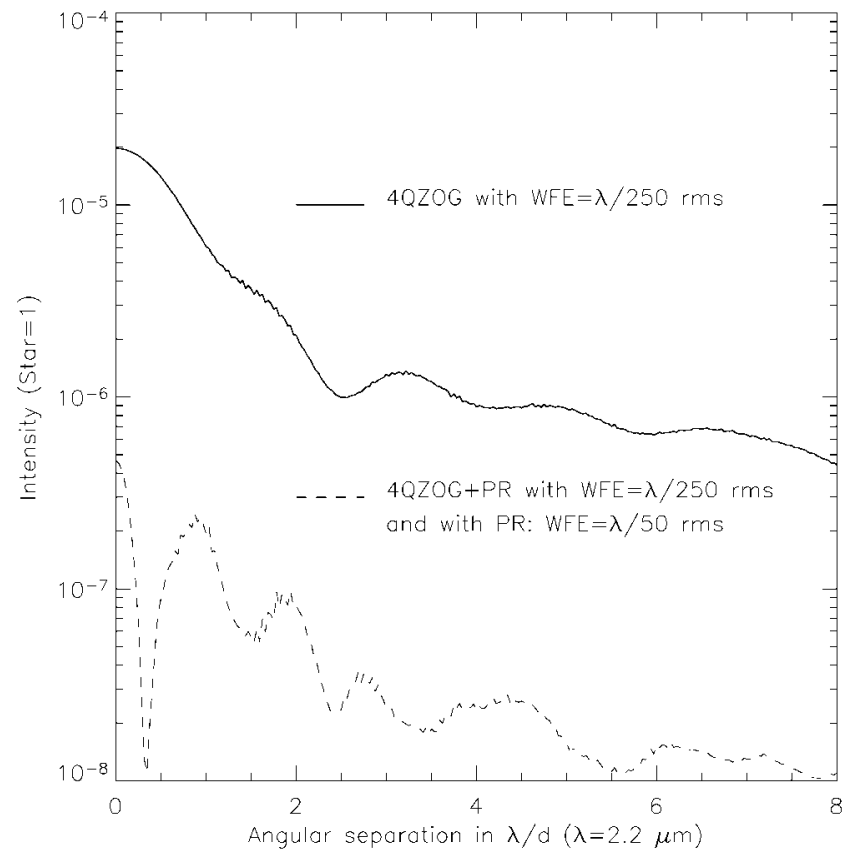

FIG. 7.-Three-stage coronagraph. Numerical simulation results for a multistage coronagraph. The first coronagraph is the quasi-achromatic 4QZOG (Mawet et al. 2005). The null depth with respect to the star is around $2 \times$ $10^{-5}$ with a wave-front error (WFE) on the entrance pupil of $\lambda / 250 \mathrm{rms}$. A second coronagraphic mask, a simple Lyot with a diameter of only $\lambda / d$, placed after a pupil replication (PR) device, gives a gain in the central stellar peak in the final image of $\approx 40$. It must be noted that the wave-front error of the replication scheme is strongly relaxed here with only $\lambda / 50 \mathrm{rms}$. For the different stages of coronagraphic devices, we choose a diaphragm of $80 \%$ of the previous pupil diameter. All wave-front errors are given for the mean wavelength of the $K_{s}$ band $(2.2 \mu \mathrm{m})$.

perimented on a coronagraphic workbench in order to prove its relevance and assess the real gain that it could provide.

We are grateful for the referee, A. Greenaway, and F. H. P. Spaan for useful discussions and suggestions, especially concerning chromatic smearing. P. R. also acknowledges the financial support of the Belgian fund "Pôle d'Attraction Interuniversitaire (PAI)."

\section{REFERENCES}

Aime, C., Soummer, R., \& Ferrari, A. 2002, A\&A, 389, 334

Baudoz, P., Rabbia, Y., \& Gay, J. 2000, A\&AS, 141, 319

Bracewell, R. N. 1978, Nature, 274, 780

Brillet, A., Vinet, J.-Y., Loriette, V., Mackowski, J.-M., Pinard, L., \& Remillieux, A. 2003, Phys. Rev. D, 67, 102006

Gillet, S., et al. 2003, A\&A, 400, 393

Goldberg, K. A., et al. 2002, J. Vac. Sci Technol. B, 20(6), 2834

Greenaway, A. H., Spaan, F. H. P., \& Mourai, V. 2005, ApJ, 618, L165 (GR05)
Labeyrie, A. 1996, A\&AS, 118, 517

Mackowski, J. M., et al. 1999, Opt. Quant. Electron., 31(5-7), 507

Mawet, D., et al. 2005, Appl. Opt., in press

Nisenson, P., \& Papaliolos, C. 2001, ApJ, 548, L201

Pedretti, E., Labeyrie, A., Arnold, L., Thureau, N., Lardiere, O., Boccaletti, A., \& Riaud, P. 2000, A\&AS, 147, 285

Riaud, P., Boccaletti, A., Baudrand, J., \& Rouan, D. 2003, PASP, 115, 712

Riaud, P., et al. 2002, A\&A, 396, 345 